\title{
A FLOATING BODY APPROACH TO FEFFERMAN'S HYPERSURFACE MEASURE
}

\author{
DAVID E. BARRETT*
}

\begin{abstract}
The floating body approach to affine surface area is adapted to a holomorphic context providing an alternate approach to Fefferman's invariant hypersurface measure.
\end{abstract}

\section{Introduction}

In [2, p. 259] Fefferman introduced a measure $\sigma_{Z}$ on an arbitrary smooth strictly pseudoconvex hypersurface $Z$ in $C^{n}$. Viewing $\sigma_{Z}$ as a positive ( $\left.2 n-1\right)$-form, it is characterized by the equation

$$
\sigma_{Z} \wedge d \rho=2^{2 n /(n+1)} M(\rho)^{1 /(n+1)} \omega_{C^{n}}
$$

where $\omega_{\mathrm{C}^{n}}$ is the euclidean volume form, $\rho$ is a defining function for $Z$ (i.e., $Z$ is the zero set of $\rho$ and the derivative of $\rho$ is positive on vectors transverse to $Z$ and pointing to the pseudoconcave side of $Z$ ), and $M$ denotes the complex Monge-Ampère operator defined by

$$
M(\rho)=(-1)^{n} \operatorname{det}\left(\begin{array}{cc}
\rho & \rho_{z_{j}} \\
\rho_{z_{\bar{k}}} & \rho_{z_{j} \bar{z}_{k}}
\end{array}\right) .
$$

(The subscripts denote differentiation.)

The interest in $\sigma_{Z}$ stems in part from the transformation law

$$
G^{*} \sigma_{G(Z)}=\left|\operatorname{det} G^{\prime}\right|^{2 n /(n+1)} \sigma_{Z}
$$

valid for $G$ biholomorphic near $Z$ (or for $G$ a CR diffeomorphism on $Z$ ).

In the case of a tube hypersurface $Z=X \times i \mathrm{R}^{n} \subset \mathrm{R}^{n} \times i \mathrm{R}^{n}=\mathrm{C}^{n}$ it is easy to check (see $\$ 2$ below) that

$$
\sigma_{Z}=\kappa_{X}^{1 /(n+1)} s_{X} \cdot \omega_{i \mathrm{R}^{n}}
$$

\footnotetext{
* This material is based upon work supported in part by the National Science Foundation under Grant No. DMS-0072237.

Received November 11, 2004.
} 
here $\omega_{i \mathrm{R}^{n}}$ is the euclidean volume form on $i \mathrm{R}^{n}, s_{X}$ is euclidean surface area on $X$, and $\kappa_{X}$ is the Gaussian curvature of $X$.

The factor $\tilde{\sigma}_{X} \stackrel{\text { def }}{=} \kappa_{X}^{1 /(n+1)} s_{X}$ above defines a measure on $X$ which has a longer history; it is the "affine surface measure" studied by Blaschke [1]. It satisfies the transformation law

$$
F^{*} \tilde{\sigma}_{F(X)}=\left|\operatorname{det} F^{\prime}\right|^{(n-1) /(n+1)} \tilde{\sigma}_{X}
$$

for $F$ affine.

In the case of $\mathrm{R}^{2}$ Blaschke provided an alternate description which applies to general convex curves. In recent years several works have provided similar results in higher dimensions. (For an overview see [8].) Some of these approaches do not seem to lend themselves to natural generalization to several complex variables, but one approach is promising for this purpose, namely that taken in papers by Leichtweiß [9] and by Schütt and Werner [12] using "floating body" theory, building on earlier work of Blaschke.

A convex body $K \subset \subset \mathrm{R}^{n}$ and a positive quantity $\delta$ determine a convex floating body defined to be the intersection of all closed half-spaces $H$ such that $K \backslash H$ has volume $\delta$. It is common to denote this object by $K_{\delta}$, but for notational convenience in this paper we will let $K_{\delta}$ denote the portion of $K$ lying outside the convex floating body.

For $n=3$ and $K$ strictly convex with analytic boundary, Blaschke showed [1] that the affine surface area of $b K$ coincides with

$$
\sqrt{\pi} \lim _{\delta \searrow 0} \frac{\operatorname{vol}\left(K_{\delta}\right)}{\sqrt{\delta}} .
$$

For general $n$ and $K$ strictly convex with $C^{2}$ boundary, Leichtweiß showed [9] that the affine surface area of $\mathrm{b} K$ coincides with

$$
\lim _{\delta \searrow 0} c_{n} \frac{\operatorname{vol}\left(K_{\delta}\right)}{\delta^{2 /(n+1)}},
$$

where

$$
c_{n}=\frac{(2 \pi)^{(n-1) /(n+1)}}{\left(\Gamma\left(\frac{n+1}{2}\right)\right)^{2 /(n+1)}} .
$$

In [12] it is shown that for any bounded convex body $K$ in $\mathrm{R}^{n}$ the limit

$$
\lim _{\delta \searrow 0} c_{n} \frac{\operatorname{vol}\left(K_{\delta}\right)}{\delta^{2 /(n+1)}}
$$

exists and is finite, coinciding with the affine surface area whenever $K$ has $C^{2}$ boundary. (See $\$ 4$ below for more on this result.) 
In this paper we provide a generalization of the results (1.6) and (1.7) to Fefferman's measure.

Let $\Omega \subset \mathrm{C}^{n}$ be a bounded strictly pseudoconvex domain with $C^{3}$ boundary. For $M>0$ let $P_{M}(\Omega)$ denote the set of $C^{3}$ functions $h$ on $\bar{\Omega}$ satisfying the conditions

(1) $h$ is holomorphic on $\Omega$;

(2) $h$ and all its derivatives of order $\leq 3$ are bounded in absolute value by $M$ on $\bar{\Omega}$;

(3) $\bar{\Omega} \cap h^{-1}(0)$ is a non-empty subset of $\mathrm{b} \Omega$;

(4) $|d h| \geq M^{-1}$ on $\bar{\Omega} \cap h^{-1}(0)$.

Note that while $P_{M}(\Omega)$ is not in general biholomorphically invariant, if $G: \bar{\Omega}_{1} \rightarrow \bar{\Omega}_{2}$ is a $C^{3}$ diffeomorphism holomorphic on $\Omega_{1}$ then for $M>0$ there are $M_{\sharp}>M_{b}>0$ so that $P_{M_{b}}\left(\Omega_{2}\right) \circ G \subset P_{M}\left(\Omega_{1}\right) \subset P_{M_{\sharp}}\left(\Omega_{2}\right) \circ G$.

For $\delta>0$ let

$$
\Omega_{M, \delta}=\bigcup_{h \in P_{M}(\Omega)}\{z \in \Omega: \operatorname{vol}(\{w \in \Omega:|h(w)| \leq|h(z)|\})<\delta\} .
$$

THEOREM 1. For $\Omega$ as above and for all $M \geq M_{0}(\Omega)$ we have

$$
C_{n} \lim _{\delta \searrow 0} \frac{\operatorname{vol}\left(\Omega_{M, \delta}\right)}{\delta^{1 /(n+1)}}=\int_{\mathrm{b} \Omega} \sigma_{\mathrm{b} \Omega},
$$

where $C_{n}$ denotes the constant

$$
\left(\frac{2^{2 n-2} \pi^{n-\frac{1}{2}} \Gamma\left(\frac{n}{2}\right)}{(n+1) \Gamma\left(\frac{n+1}{2}\right) \Gamma(n)}\right)^{\frac{1}{n+1}} .
$$

This theorem will be proved in $\S 3$. $\$ 2$ has more information concerning the construction of Fefferman's measure. The final section lays out some open questions.

It may strike some readers at this stage that when generalizing the floating body construction to the holomorphic setting it would seem natural to focus on sublevel sets of $\operatorname{Re} h$ rather than $|h|$. Let us address this first in the onedimensional setting, where $\sigma_{\mathrm{b} \Omega}$ is the standard element of arc length $|d z|$ (see $\$ 2$ below). Attempts to understand the volume of small sublevel sets $\operatorname{Re} h$ lead to consideration of second-order information about $\mathrm{b} \Omega$ - but such information simply doesn't appear in the integral $\int_{\mathrm{b} \Omega} \sigma_{\mathrm{b} \Omega}=$ length $(\mathrm{b} \Omega)$. But for $h \in P_{M}(\Omega)$ and $\epsilon>0$ small the set $\Omega \cap|h|^{-1}([0, \epsilon])$ is approximately a half-disk, and the parameter $M$ gives us enough uniformity to assert that for 
small $\delta>0$ the set $\Omega_{M, \delta}$ is a collar about $\mathrm{b} \Omega$ of normal width approximately $\sqrt{2 \delta / \pi}$, hence

$$
\sqrt{\pi / 2} \lim _{\delta \searrow 0} \frac{\operatorname{vol}\left(\Omega_{M, \delta}\right)}{\sqrt{\delta}}=\text { length }(\mathrm{b} \Omega)
$$

as claimed in the theorem.

In higher dimensions the focus on $|h|$ rather than $\operatorname{Re} h$ allows us to restrict our consideration of second-order information to the complex directions in the tangent spaces of $b \Omega$. A related point is that the small sublevel sets of $|h|$ reflect the non-isotropic structure of $b \Omega$ (see for example $[11, \S 5.1]$ ).

\section{On the construction of Fefferman's measure}

Let $Z$ be a $C^{2}$ strictly pseudoconvex hypersurface in an $n$-dimensional complex manifold $M$ equipped with a smooth positive $2 n$-form $\omega$. We will explain how to construct a positive $(2 n-1)$-form $\sigma_{Z, \omega}$ on $Z$ in such a way that the transformation law

$$
G^{*} \sigma_{G(Z), \tilde{\omega}}=\left(\frac{G^{*} \tilde{\omega}}{\omega}\right)^{n /(n+1)} \sigma_{Z, \omega}
$$

holds for $G$ biholomorphic.

Let $J$ denote the complex structure tensor (thus in $\mathrm{C}^{n}$ we have $J \frac{\partial}{\partial x_{j}}=\frac{\partial}{\partial y_{j}}$, $\left.J \frac{\partial}{\partial y_{j}}=-\frac{\partial}{\partial x_{j}}\right)$.

The Levi-form $\mathscr{L}$ of $Z$ may be naturally defined as a symmetric $T M / T Z$ valued form on $T Z \cap J T Z$ characterized by the identity

$$
\mathscr{L}\left(Y_{1}, Y_{2}\right) \equiv\left[Y_{1}, J Y_{2}\right] \quad \bmod T Z \cap J T Z
$$

for $T Z \cap J T Z$-valued vector fields $Y_{1}$ and $Y_{2}$. The Levi-form is (real-)hermitian (i.e., $\left.\mathscr{L}\left(J Y_{1}, J Y_{2}\right)=\mathscr{L}\left(Y_{1}, Y_{2}\right)\right)$. (The hermitian property follows directly from the integrability condition $J\left(\left[Y_{1}, Y_{2}\right]-\left[J Y_{1}, J Y_{2}\right]\right)=\left[J Y_{1}, Y_{2}\right]+$ $\left[Y_{1}, J Y_{2}\right]$; the symmetry of $\mathscr{L}$ follows from the hermitian property and the antisymmetry of the bracket operation.) Note also that $\mathscr{L}(Y, J Y)=0$.

We carry out the construction first in the two-dimensional case.

Let $Y$ be a non-zero vector in $T Z \cap J T Z$. Then $Y, J Y, \mathscr{L}(Y, Y)$ gives a basis for $T Z$. We describe $\sigma_{Z, \omega}$ by the identity

$$
\sigma_{Z, \omega}(Y, J Y, \mathscr{L}(Y, Y))=\omega^{2 / 3}(Y, J Y, \mathscr{L}(Y, Y), J \mathscr{L}(Y, Y)) .
$$

(We assume here that orientations have been chosen so that $Y, J Y, \mathscr{L}(Y, Y)$ and $Y, J Y, \mathscr{L}(Y, Y), J \mathscr{L}(Y, Y)$ are positive bases for $T Z$ and $T M$ respectively.)

If $Y$ is replaced by $\tilde{Y}=\alpha Y+\beta J Y$ then both sides of (2.1) pick up a factor of $\left(\alpha^{2}+\beta^{2}\right)^{2}$; it follows that $\sigma_{Z, \omega}$ does not depend on the choice of $Y$. 
In higher dimension we choose a complex basis $Y_{1}, \ldots, Y_{n-1}$ of $T Z \cap J T Z$. Let $L_{j, k}=\mathscr{L}\left(Y_{j}, Y_{k}\right)-i \mathscr{L}\left(J Y_{j}, Y_{k}\right)$. (Thus $\left(L_{j, k}\right)$ is the (complex-)hermitian matrix representing $\mathscr{L}$ with respect to the given basis.) Using the Levi-form to orient $T M / T Z$, note that $\operatorname{det}^{1 /(n-1)}\left(L_{j, k}\right)$ defines a vector in $T M / T Z$. We then describe $\sigma_{Z, \omega}$ by the identity

$$
\begin{aligned}
& \sigma_{Z, \omega}\left(Y_{1}, J Y_{1}, \ldots, Y_{n-1}, J Y_{n-1}, \operatorname{det}^{\frac{1}{n-1}}\left(L_{j, k}\right)\right) \\
& \quad=\omega^{\frac{n}{n+1}}\left(Y_{1}, J Y_{1}, \ldots, Y_{n-1}, J Y_{n-1}, \operatorname{det}^{\frac{1}{n-1}}\left(L_{j, k}\right), J \operatorname{det}^{\frac{1}{n-1}}\left(L_{j, k}\right)\right) .
\end{aligned}
$$

If $Y_{1}, \ldots, Y_{n-1}$ are replaced by $\sum \alpha_{1, k} Y_{k}+\sum \beta_{1, k} J Y_{k}, \ldots, \sum \alpha_{n-1, k} Y_{k}+$ $\sum \beta_{n-1, k} J Y_{k}$, (with $\alpha_{j, k}$ and $\beta_{j, k}$ real) then both sides of (2.2) pick up a factor of $|\operatorname{det}(\alpha+i \beta)|^{2 n /(n-1)}$; as before if follows that $\sigma_{Z, \omega}$ does not depend on the choice of $Y_{1}, \ldots, Y_{n-1}$.

We claim that for $M=\mathrm{C}^{n}$ equipped with the euclidean volume form $\omega$ the form $\sigma_{Z, \omega}$ defined in (2.2) coincides with the form $\sigma_{Z}$ defined in (1.2). It will suffice to check this at the origin under that assumption that $\rho$ is locally of the form $\psi\left(z_{1}, \ldots, z_{n-1}, \operatorname{Re} z_{n}\right)-\operatorname{Im} z_{n}$ with $\psi$ and its gradient vanishing at 0 . Then

$$
\sigma_{Z}=2^{\frac{2 n}{n+1}} M(\rho)^{\frac{1}{n+1}} d x_{1} \wedge d y_{1} \ldots \wedge d x_{n-1} \wedge d y_{n-1} \wedge d x_{n}
$$

at 0 ; setting $Y_{j}=4 \operatorname{Re}\left(\frac{\partial \rho}{\partial z_{n}} \frac{\partial}{\partial z_{j}}-\frac{\partial \rho}{\partial z_{j}} \frac{\partial}{\partial z_{n}}\right), 1 \leq j \leq n-1$, in (2.2) and checking that $L_{j, k}=4 \rho_{z_{j}, \bar{z}_{k}} \cdot \frac{\partial}{\partial x_{n}}$ and $\operatorname{det}\left(L_{j, k}\right)=2^{2 n} M(\rho)\left(\frac{\partial}{\partial x_{n}}\right)^{n-1}$ at 0 we have

$$
\begin{aligned}
2^{\frac{2 n}{n-1}} M(\rho)^{\frac{1}{n-1}} \sigma_{Z, \omega}\left(\frac{\partial}{\partial x_{1}}, \frac{\partial}{\partial y_{1}}, \ldots, \frac{\partial}{\partial x_{n-1}}, \frac{\partial}{\partial y_{n-1}},\right. & \left.\frac{\partial}{\partial x_{n}}\right) \\
& =2^{\frac{4 n}{n-1} \frac{n}{n+1}} M(\rho)^{\frac{2}{n-1} \frac{n}{n+1}}
\end{aligned}
$$

at 0 ; with a little further manipulation of exponents we find that $\sigma_{Z, \omega}=\sigma_{Z}$ as claimed.

Note that using (2.3) we may write $\sigma_{Z}$ in completely euclidean terms - up to a multiplicative constant - as $|\operatorname{det} \mathscr{L}|^{1 /(n+1)} s_{Z}$, where $s_{Z}$ is the euclidean surface area on $Z$ and the bars on $|\operatorname{det} \mathscr{L}|$ indicate measurement with respect to the euclidean structure. In the case of a tube domain $Z=X \times i \mathrm{R}^{n}, \mathscr{L}$ is essentially just the second fundamental form of $X$, so $|\operatorname{det} \mathscr{L}|$ is just the Gaussian curvature of $X$. To check that no multiplicative constant is missing from (1.4) one can trace through the construction or test both sides against the hypersurface $\left\{\left(z_{1}, \ldots, z_{n}\right): x_{1}^{2}+\cdots+x_{n}^{2}=1\right\}$. (Remark: In [2], Fefferman allows a dimension-dependent constant factor in the definition of $\sigma_{Z}$; we have 
chosen the constant $2^{(2 n+1) /(n+1)}$ in (1.1) to arrange that (1.4) holds. A different choice appears in [5].)

For $n=1$ many of the above computations are problematic, but we can see that in this case the natural analogue of the above construction is given by the formula

$$
\sigma_{Z, \omega}(Y)=\omega^{1 / 2}(Y, J Y)
$$

converting a positive area form on $M$ to a positive one-form on $Z$. In particular, for $M=C$ equipped with the euclidean area form $\omega, \sigma_{Z, \omega}$ is the standard arc length form, agreeing with $\sigma_{Z}$ given by (1.1).

\section{Proof of main theorem}

Fix for the moment a function $h \in P_{M}(\Omega)$ and a point $p \in \mathrm{b} \Omega$ where $h$ vanishes.

Choose a unitary system of coordinates $\left(w_{1}, \ldots, w_{n}\right)$ vanishing at $p$ so that the tangent space to $\mathrm{b} \Omega$ is given by $\operatorname{Im} w_{n}=0$. Since the zero set of $h$ must be tangent to $\mathrm{b} \Omega$, we have $d h=h_{w_{n}} d w_{n}$ at 0 . Replacing $h$ by $h_{w_{n}}^{-1}(0) \cdot h$ we may assume that $d h=d w_{n}$ at 0 , this at the cost of squaring $M$.

Let $\gamma$ be the local solution to the ordinary differential equation

$$
\gamma_{w_{1}} h_{w_{n}}-\gamma_{w_{n}} h_{w_{1}}=1
$$

subject to the initial condition

$$
\gamma\left(0, w_{2}, \ldots, w_{n}\right)=0 .
$$

Then the functions $z_{1}, \ldots, z_{n}$ defined by

$$
\begin{aligned}
& z_{1}=\gamma(w) \\
& z_{j}=w_{j} \text { for } 2 \leq j \leq n-1 \\
& z_{n}=h(w)
\end{aligned}
$$

define a volume-preserving holomorphic change of coordinates near $p$.

Note that $\frac{\partial z_{j}}{\partial w_{k}}(0)=\delta_{j, k}$; thus this change of coordinates preserves distances up to a factor of $1+O(\|z\|)$.

With this set up we wish to study the volumes of the sets

$$
S_{\eta} \stackrel{\text { def }}{=}\left\{z \in \Omega:\left|z_{n}\right|<\eta\right\}
$$

where $\Omega$ is locally described by an inequality

$$
\operatorname{Im} z_{n}>\psi\left(z_{1}, \ldots, z_{n-1}, \operatorname{Re} z_{n}\right)
$$


and satisfies

$$
z_{n} \neq 0 \text { in } \Omega \text {. }
$$

Let us focus for the time being on the case $n=2$. Set $z_{1}=z, z_{2}=u+i v$. Then we may write

$$
\psi(z, u)=\lambda(u)|z|^{2}+\operatorname{Re} \mu(u) z^{2}+\operatorname{Re} v(u) z+\xi(u)+O\left(|z|^{3}\right)
$$

with $v(0)=\xi(0)=\xi^{\prime}(0)=0$.

The strict pseudoconvexity of $\Omega$ implies that $\lambda(0)>0$ and condition (3.1) implies that $|\mu(0)| \leq \lambda(0)$

Let $\tilde{\lambda}=\sqrt{\lambda^{2}+|\mu|^{2}}$. Note that $2|\mu(0)|^{2} \leq \lambda(0)^{2}+|\mu(0)|^{2}=\tilde{\lambda}^{2}(0)$, so

$$
|\mu(0)| \leq \frac{1}{\sqrt{2}} \tilde{\lambda}(0)
$$

Let

$$
\begin{aligned}
\tilde{\psi}(z, u) & =\psi(z, u)+(\tilde{\lambda}(u)-\lambda(u))|z|^{2} \\
& =\tilde{\lambda}(u)|z|^{2}+\operatorname{Re} \mu(u) z^{2}+\operatorname{Re} v(u) z+\xi(u)+O\left(|z|^{3}\right),
\end{aligned}
$$

and let $\tilde{\Omega} \subset \Omega$ be a domain defined near $p$ by the inequality $v>\tilde{\psi}(z, u)$.

For $\eta$ small we may use (3.3) and (3.4) to conclude that on $b \tilde{\Omega} \cap S_{\eta}$ we have

$$
\begin{aligned}
\left(1-\frac{1}{\sqrt{2}}\right) \tilde{\lambda}(0)|z|^{2} & \leq \tilde{\lambda}(0)|z|^{2}+\operatorname{Re} \mu(0) z^{2} \\
& =v+O(\eta(\eta+|z|))+O\left(|z|^{3}\right)
\end{aligned}
$$

thus $|z|^{2}=O(\eta(1+|z|))$ and $|z|=O(\sqrt{\eta})$, so $\tilde{\lambda}(0)|z|^{2}+\operatorname{Re} \mu(0) z^{2}=$ $v+O\left(\eta^{3 / 2}\right)$.

Quoting the fact that $\left\{z: A|z|^{2}+\operatorname{Re} B z^{2}<V\right\}$ has area equal to $\frac{\pi V^{+}}{\sqrt{A^{2}-|B|^{2}}}$ when $|B|<A$ we find that $\{z:(z, u+i v) \in \tilde{\Omega}\}$ has area equal to

$$
\frac{\pi v^{+}+O\left(\eta^{3 / 2}\right)}{\sqrt{\tilde{\lambda}^{2}(0)-|\mu(0)|^{2}}}=\frac{\pi v^{+}+O\left(\eta^{3 / 2}\right)}{\lambda(0)} .
$$


Thus

$$
\begin{aligned}
\operatorname{vol}(\{(z, u+i v) \in \Omega:|u+i v|<\eta\}) \\
\geq \operatorname{vol}(\{(z, u+i v) \in \tilde{\Omega}:|u+i v|<\eta\}) \\
=\iint_{u^{2}+v^{2}<\eta^{2}} \frac{\pi v^{+}+O\left(\eta^{3 / 2}\right)}{\lambda(0)} d u d v \\
=\int_{0}^{2 \pi} \int_{0}^{\eta} \frac{\pi r \sin ^{+} \theta+O\left(\eta^{3 / 2}\right)}{\lambda(0)} r d r d \theta \\
=\frac{2 \pi \eta^{3}+O\left(\eta^{7 / 2}\right)}{3 \lambda(0)}=\frac{8 \pi \eta^{3}+O\left(\eta^{7 / 2}\right)}{3\left(\frac{\sigma_{Z}}{s_{Z}}(0)\right)^{3}} .
\end{aligned}
$$

The above estimates are uniform in $p$ and show that $\Omega_{M, \delta}$ is contained in a collar about $\mathrm{b} \Omega$ of normal thickness $\left(\left(\frac{3 \delta}{8 \pi}\right)^{1 / 3}+O\left(\delta^{1 / 2}\right)\right) \frac{\sigma_{Z}}{s_{Z}}(0)$.

Thus

$$
\left(\frac{8 \pi}{3}\right)^{1 / 3} \limsup _{\delta \searrow 0} \frac{\operatorname{vol}\left(\Omega_{M, \delta}\right)}{\delta^{1 / 3}} \leq \int_{\mathrm{b} \Omega} \sigma_{\mathrm{b} \Omega}
$$

To get an estimate in the other direction we make use of that fact that when $M$ is large enough, for each $p \in \mathrm{b} \Omega$ we can find $h_{p} \in P_{M}(\Omega)$ such that

- $\bar{\Omega} \cap h_{p}^{-1}(0)=\{p\}$;

- $\left\|d h_{p}(p)\right\|=1$;

- $\Omega \cap\left|h_{p}\right|^{-1}([0, \epsilon])=\left\{z \in \Omega:\left|h_{p}(z)\right| \leq \epsilon\right\}$ is connected for $\epsilon<\epsilon_{0}$ (with $\epsilon_{0}$ independent of $p$ );

- after introducing new coordinates as above we have $\mu(0)=0$.

(See [4, §2.4], [7, §5.2].) Then (3.5) can be revised to read

$$
\operatorname{vol}(\{(z, u+i v) \in \Omega:|z|<\eta\})=\frac{8 \pi \eta^{3}+O\left(\eta^{7 / 2}\right)}{3\left(\frac{\sigma_{Z}}{s_{Z}}(0)\right)^{3}} .
$$

As above, it follows that $\Omega_{M, \delta}$ contains a collar about $\mathrm{b} \Omega$ of normal thickness $\left(\left(\frac{3 \delta}{8 \pi}\right)^{1 / 3}+O\left(\delta^{1 / 2}\right)\right) \frac{\sigma_{Z}}{s_{Z}}(0)$, implying that

$$
\left(\frac{8 \pi}{3}\right)^{1 / 3} \liminf _{\delta \searrow 0} \frac{\operatorname{vol}\left(\Omega_{M, \delta}\right)}{\delta^{1 / 3}} \geq \int_{\mathrm{b} \Omega} \sigma_{\mathrm{b} \Omega} .
$$


Combining (3.6) and (3.7) we have (1.10) in the case $n=2$.

To treat the case $n>2$ we modify the argument as follows. We now set $\left(z_{1}, \ldots, z_{n-1}\right)=z^{\prime}, z_{n}=u+i v$. The expansion (3.2) now reads

$$
\begin{aligned}
\psi\left(z^{\prime}, u\right)=\sum_{j, k=1}^{n-1} \lambda_{j, k}(u) z_{j} \bar{z}_{k}+\operatorname{Re} \sum_{j, k=1}^{n-1} \mu_{j, k}(u) z_{j} z_{k} & \\
& +\operatorname{Re} \sum_{j=1}^{n-1} \nu_{j}(u) z_{j}+\xi(u)+O\left(\left\|z^{\prime}\right\|^{3}\right)
\end{aligned}
$$

with $v_{j}(0)=\xi(0)=\xi^{\prime}(0)=0, \lambda_{k, j}=\bar{\lambda}_{j, k}, \mu_{k, j}=\mu_{j, k}$.

We may choose an invertible linear map $T=\left(T_{1}, \ldots, T_{n-1}\right): \mathrm{C}^{n-1} \rightarrow$ $\mathrm{C}^{n-1}$ and $\phi_{j} \geq 0$ so that

$$
\psi\left(z^{\prime}, 0\right)=\sum_{j=1}^{n-1}\left(\left|T_{j} z^{\prime}\right|^{2}+\operatorname{Re} \phi_{j}\left(T_{j} z^{\prime}\right)^{2}\right)+O\left(\left\|z^{\prime}\right\|^{3}\right) .
$$

(See for example Lemma 4.1 in [13].)

Condition (3.1) implies that each $\phi_{j} \leq 1$.

Let $\left(\tilde{\lambda}_{j, k}\right)$ be the hermitian matrix satisfying

$$
\sum_{j, k=1}^{n-1} \tilde{\lambda}_{j, k} z_{j} \bar{z}_{k}=\sum_{j=1}^{n-1} \sqrt{1+\phi_{j}^{2}}\left|T_{j} z^{\prime}\right|^{2} .
$$

In analogy to (3.3) we have

$$
\operatorname{Re} \sum_{j, k=1}^{n-1} \mu_{j, k}(0) z_{j} z_{k} \leq \frac{1}{\sqrt{2}} \sum_{j, k=1}^{n-1} \tilde{\lambda}_{j, k} z_{j} \bar{z}_{k} .
$$

We now let

$$
\begin{gathered}
\tilde{\psi}\left(z^{\prime}, u\right)=\psi\left(z^{\prime}, u\right)+\sum_{j, k=1}^{n-1}\left(\tilde{\lambda}_{j, k}-\lambda_{j, k}(u)+O(|u|)\right) z_{j} \bar{z}_{k} \\
=\sum_{j, k=1}^{n-1} \tilde{\lambda}_{j, k} z_{j} \bar{z}_{k}+\operatorname{Re} \sum_{j, k=1}^{n-1} \mu_{j, k}(u) z_{j} z_{k} \\
\quad+\operatorname{Re} \sum_{j=1}^{n-1} v_{j}(u) z_{j}+\xi(u)+O\left(\left\|z^{\prime}\right\|^{3}\right),
\end{gathered}
$$


where the $O(|u|)$ term is chosen so that there is a domain $\tilde{\Omega} \subset \Omega$ defined near $p$ by $v>\tilde{\psi}\left(z^{\prime}, u\right)$. On b $\tilde{\Omega} \cap S_{\eta}$ we have as before $\left\|z^{\prime}\right\|=O(\sqrt{\eta})$, and

$$
\sum_{j, k=1}^{n-1} \tilde{\lambda}_{j, k} z_{j} \bar{z}_{k}+\operatorname{Re} \sum_{j, k=1}^{n-1} \mu_{j, k}(0) z_{j} z_{k}=v+O\left(\eta^{3 / 2}\right) .
$$

The set

$$
\begin{aligned}
\left\{z^{\prime}: \sum_{j, k=1}^{n-1} \tilde{\lambda}_{j, k} z_{j} \bar{z}_{k}+\operatorname{Re}\right. & \left.\sum_{j, k=1}^{n-1} \mu_{j, k}(0) z_{j} z_{k}<V\right\} \\
& =\left\{z^{\prime}: \sum_{j=1}^{n-1} \sqrt{1+\phi_{j}^{2}}\left|T_{j} z^{\prime}\right|^{2}+\operatorname{Re} \phi_{j}\left(T_{j} z^{\prime}\right)^{2}<V\right\}
\end{aligned}
$$

has volume

$$
\begin{array}{r}
|\operatorname{det} T|^{-2} \prod_{j=1}^{n-1} \frac{1}{\sqrt{1+\phi_{j}^{2}}+\phi_{j}} \frac{1}{\sqrt{1+\phi_{j}^{2}}-\phi_{j}} \cdot \operatorname{vol}\left(\left\{z^{\prime}:\left\|z^{\prime}\right\|<V\right\}\right) \\
=\frac{\pi^{n-1}\left(V^{+}\right)^{n-1}}{(n-1) !|\operatorname{det} T|^{2}}
\end{array}
$$

thus

$$
\begin{aligned}
& \operatorname{vol}\left(\left\{\left(z^{\prime}, u+i v\right) \in \Omega:|u+i v|<\eta\right\}\right) \\
& \quad \geq \operatorname{vol}\left(\left\{\left(z^{\prime}, u+i v\right) \in \tilde{\Omega}:|u+i v|<\eta\right\}\right) \\
& \quad=\iint_{u^{2}+v^{2}<\eta^{2}} \frac{\pi^{n-1}\left(v^{+}\right)^{n-1}+O\left(\eta^{n-\frac{1}{2}}\right)}{(n-1) !|\operatorname{det} T|^{2}} d u d v \\
& \quad=\int_{0}^{2 \pi} \int_{0}^{\eta} \frac{\pi^{n-1} r^{n-1}\left(\sin ^{+} \theta\right)^{n-1}+O\left(\eta^{n-\frac{1}{2}}\right)}{(n-1) !|\operatorname{det} T|^{2}} r d r d \theta \\
& \quad=\frac{\pi^{n-\frac{1}{2}} \eta^{n+1} \Gamma\left(\frac{n}{2}\right)+O\left(\eta^{\eta+\frac{3}{2}}\right)}{(n+1) \Gamma\left(\frac{n+1}{2}\right) \Gamma(n)|\operatorname{det} T|^{2}}=\frac{2^{2 n-2} \pi^{n-\frac{1}{2}} \eta^{n+1} \Gamma\left(\frac{n}{2}\right)+O\left(\eta^{\eta+\frac{3}{2}}\right)}{(n+1) \Gamma\left(\frac{n+1}{2}\right) \Gamma(n)\left(\frac{\sigma_{Z}}{s_{Z}}(0)\right)^{n+1}} .
\end{aligned}
$$

Using this estimate as before we find that

$$
C_{n} \limsup _{\delta \searrow 0} \frac{\operatorname{vol}\left(\Omega_{M, \delta}\right)}{\delta^{1 /(n+1)}} \leq \int_{\mathrm{b} \Omega} \sigma_{\mathrm{b} \Omega} .
$$


Quoting as before the existence of peaking functions $h_{p}$ based on the Levi polynomial we get the complementary estimate

$$
C_{n} \liminf _{\delta \searrow 0} \frac{\operatorname{vol}\left(\Omega_{M, \delta}\right)}{\delta^{1 /(n+1)}} \geq \int_{\mathrm{b} \Omega} \sigma_{\mathrm{b} \Omega} .
$$

Combining the estimates we have (1.10).

\section{Comments}

(1) The proof of Theorem 1 can easily be adapted to yield the following result:

THeOREM 2 . Let $\Omega$ be a relatively compact strictly pseudoconvex domain with $C^{3}$ boundary inside a complex manifold equipped with a smooth positive $2 n$-form $\omega$. Let $\Omega_{M, \delta, \omega}$ be defined as in (1.9). Then

$$
C_{n} \lim _{\delta \searrow 0} \frac{\operatorname{vol}_{\omega}\left(\Omega_{M, \delta, \omega}\right)}{\delta^{1 /(n+1)}}=\int_{\mathrm{b} \Omega} \sigma_{\mathrm{b} \Omega, \omega} .
$$

(2) It would be interesting to know if a result like Theorem 1 holds also for weakly pseudoconvex domains (possibly involving some reformulation of the family $P_{M}(\Omega)$ ), and if a limit like (1.10) can be shown to exist (independent of the choice of $M \geq M_{0}$ ) also in non-smooth settings.

Note that in the case of a polydisk, the limit (1.10) does exist and in fact it vanishes.

(3) For a general bounded convex body $K$ in $\mathrm{R}^{n}$ it is known that the limit (1.8) coincides with the integral

$$
\int_{\mathrm{b} K} \kappa_{\mathrm{b} K}^{1 /(n+1)} s_{\mathrm{b} K},
$$

where $s_{\mathrm{b} K}$ denotes $(n-1)$-dimensional Hausdorff measure and $\kappa_{\mathrm{b} K}$ denotes the Gaussian curvature of $b \kappa$ which exists in a suitable pointwise sense almost everywhere with respect to $s_{\mathrm{b} K}$ (so in essence the singular part of the curvature is discarded). (See [12], [8, §2.7].)

In the holomorphic setting there is no evident way to similarly interpret Fefferman's measure on boundaries of arbitrary bounded pseudoconvex domains. But if we impose additional hypotheses such an interpretation may be possible. It would be interesting to know if this can be carried out in particular for domains with Lipschitz boundary which admit strictly plurisubharmonic defining functions.

(4) A number of results have been proved relating affine surface area to the complexity of approximating polytopes (see the survey [3]). It would be 
interesting to have similar results in the holomorphic setting concerning approximation by analytic polyhedra. (Some natural-sounding notions of complexity of analytic polyhedra definitely will not work for this purpose: see [6, Lemma 5.3.8].)

\section{REFERENCES}

1. Blaschke, W., Vorlesungen über Differentialgeometrie II: Affine Differentialgeometrie, Springer, 1923.

2. Fefferman, C., Parabolic invariant theory in complex analysis, Adv. Math. 31 (1979), 131262.

3. Gruber, P. M., Aspects of approximation of convex bodies, in Handbook of convex geometry, Vol. A, B, 319-345, North-Holland, Amsterdam, 1993.

4. Henkin G., and Leiterer, J., Theory of Functions on Complex Manifolds, Birkhäuser, 1984.

5. Hirachi, K., Transformation law for the Szegö projectors on CR manifolds, Osaka J. Math. 27 (1990), 301-308.

6. Hörmander, L., An Introduction to Complex Analysis in Several Variables, 3rd ed., NorthHolland, 1990.

7. Krantz, S., Function Theory of Several Complex Variables (2nd ed.), Wadsworth \& Brooks/Cole, 1992.

8. Leichtweiß, K., Affine Geometry of Convex bodies, Johann Ambrosius Barth Verlag, 1998.

9. Leichtweiß, K., Über eine Formel Blaschkes zur Affinoberfläche, Studia Sci. Math. Hungar. 21 (1986), 453-474.

10. Lutwak, E., Extended affine surface area, Adv. Math. 85 (1991), 39-68.

11. Rudin, W., Function Theory in the Unit Ball of $\mathrm{C}^{n}$, Springer-Verlag, 1980.

12. Schütt C., and Werner, E., The convex floating body, Math. Scand. 66 (1990), 275-290.

13. Webster, S., On the mapping problem for algebraic real hypersurfaces, Invent. Math. 43 (1977), 53-68.

DEPT. OF MATHEMATICS

UNIVERSITY OF MICHIGAN

ANN ARBOR, MI 48109-1109

USA

E-mail: barrett@umich.edu 